\title{
Pronto atendimento infantil: quem utiliza e por que motivo
}

\author{
Marina Lucca Santos* Roger Bagolin da Silva** Maria Saleti Lock \\ Vogt $^{* * *}$ Leris Salete Bonfanti Haeffner ${ }^{\star * * *}$ Márcia Regina Canto \\ Michelotti ${ }^{\star * \star * *}$
}

Resumo: 0 objetivo deste estudo foi verificar o perfil da população pediátrica que recorre ao Pronto Atendimento Municipal Infantil (PAl), de seus acompanhantes, identificando sintomas, diagnóstico, motivo da utilização e as unidades básicas referência dos usuários. Estudo transversal descritivo realizado de julho a dezembro de 2011. Os dados foram coletados da ficha de atendimento médico e questionário aplicado aos acompanhantes. A maioria das crianças era do sexo feminino $(53,4 \%)$, com menos de 5 anos $(62,3 \%)$ e de cor branca $(78,5 \%)$. As queixas principais foram tosse $(32 \%)$ e febre $(30 \%)$ com diagnóstico de IVAS $(42,9 \%)$ e asma $(10,1 \%)$. Os motivos que determinaram a não procura pelas UBS foram falta de fichas $(44,5 \%)$, de pediatra $(20,7 \%)$ e preferência pelo PAI $(18,2 \%)$. Concluise que os atendimentos no PAI se deram por dificuldade de acesso nas UBS, em crianças de até5 anos, levados pelas mães, apresentando principalmente tosse, febre e doenças respiratórias aguda.

Descritores: Atenção Primária à Saúde; Serviços Médicos de Emergência; Acesso aos Serviços de Saúde.

\section{Emergency care for children: who uses and why}

Abstract: The aim of this study was to characterize the profile of the pediatric population that uses the Municipal Emergency Care for Children, their caregivers, identifying symptoms, diagnosis, reasons for use, and basic units of reference. Cross-sectional study conducted from July to December 2011. Data were collected from the medical record and questionnaire administered to caregivers. Most of the children were female $(53.4 \%)$, less than 5 years $(62.3 \%)$ and Caucasian $(78.5 \%)$. The main complaints were cough $(32 \%)$ and fever $(30 \%)$ diagnosed with infections of the upper airways $(42.9 \%)$ and asthma $(10.1 \%)$. The reasons for no use of basic units were lack of records $(44.5 \%)$, lack of pediatric $(20.7 \%)$ and preference for Municipal Emergency (18.2\%). We conclude that the Municipal Emergency Care for Children attendances were due to difficulty of access in basic units, in children with less than five years, brought by mothers, presenting mainly cough, fever and acute respiratory diseases.

Descriptors: Primary Health Care;Emergency Medical Services;Health Services Acessibility.

\footnotetext{
* Graduanda em Medicina na Universidade Federal de Santa Maria (UFSM), Santa Maria, RS, Brasil.

**Graduando de Medicina na Universidade Federal de Santa Maria (UFSM), Santa Maria, RS, Brasil.

***Doutora em Ciências da Saúde pela Universidade de Brasília (UNB), Brasilia, DF, Brasil.

****Doutora em Medicina pela Universidade de São Paulo (USP), Ribeirão Preto, SP, Brasil.

${ }^{* * * * *}$ Graduada em Medicina pela Universidade Federal de Santa Maria (UFSM) Santa Maria, RS, Brasil.
} 


\section{Introdução}

As unidades de Pronto Atendimento são estruturas de complexidade intermediária entre as unidades básicas de saúde e as portas de urgência hospitalares. Tais unidades servem para 0 atendimento de urgências de menor complexidade devendo referenciar ao hospital as situações clínicas de maior complexidade ${ }^{1}$. Estes serviços de urgência estão pensados para proporcionar assistência médica descontínua e concreta ficando a cargo das unidades básicas uma assistência contínua através da orientação, prevenção e quando necessário 0 encaminhamento? ${ }^{2}$.

No entanto, não só no Brasil como em outros países têm ocorrido, principalmente durante os últimos anos, um aumento progressivo na demanda de assistência nos serviços de urgência tanto gerais quanto pediátricos ${ }^{3}$.Este recrudescimento se deve preferencialmente a patologias banais estimadas por alguns autores entre um e dois terços do total de consultas ${ }^{4-}$ ${ }^{6}$. Demonstrando que o Pronto Atendimento tem se tornado importante porta de entrada ao sistema de saúde descaracterizando a missão no atendimento das urgências ${ }^{5,7}$.

Um dos principais fatores implicados nesta utilização inadequada dos serviços de urgência é a assistência nas Unidades Básicas de Saúde (UBS) e Centros de Saúde (CS), que de modo geral são muitas vezes insuficientes para dar conta da demanda ou são ineficientes e de difícil acess $0^{8}$. Outro importante fator é o usuário que, historicamente, prefere - Pronto Atendimento porque relaciona uma imagem de melhor acessibilidade e resolubilidade ao serviço porque a oferta é, na maioria das vezes, restrita no serviço de saúde primário ou por desconhecimento da legitima função do Pronto Atendimento ou porque ele realmente acredita que seu caso é urgente por desconhecer o conceito de urgência ${ }^{4-6,9}$.

Assim, pelos mais diversos motivos existe uma procura acentuada dos serviços de urgência e emergência trazendo duas consequências imediatas. A primeira 0 aumento dos custos econômicos e a segunda a necessidade de maiores recursos humanos que na maioria dos locais são insuficientes, uma vez que ficam ocupados na resolução de patologias banais, dificultando a assistência a situações verdadeiramente graves8. Desse modo, este estudo teve como objetivo verificar o perfil da população pediátrica que recorre ao Pronto Atendimento Municipal Infantil (PAl) e de seus acompanhantes, os sinais e sintomas apresentados, o diagnóstico estabelecido e os motivos de sua utilização, além de identificar as unidades básicas de saúde de referência dos usuários.

Saúde (Santa Maria), v.39, n.2, p.79-88, 2013. Pronto atendimento infantil: Quem usa e por que

\section{Metodologia}

Este estudo transversal e descritivo dos atendimentos realizados no Pronto atendimento Infantil da cidade de Santa Maria/RS foi realizado de julho a dezembro de 2011. 0 estudo foi aprovado pelo Comitê de Ética em Pesquisa da Universidade Federal de Santa Maria, dia 14/06/2011, com CAAE (Certificado de Apresentação para Apreciação Ética) de número 0146.0.243.000-11. A amostra foi de conveniência, incluindo os pacientes, crianças e/ou menores de 18 anos, atendidos em dois dias de cada semana, durante os horários em que estavam em funcionamento as Unidades Básicas de Saúde de referência dos pacientes.

A coleta de dados foi realizada em duas etapas, a primeira no momento que antecedia a consulta médica e a segunda logo após a mesma. Na sala de espera, um questionário foi 
aplicado aos cuidadores dos pacientes que aceitaram participar da pesquisa e mediante a assinatura do Termo de Consentimento Livre e Esclarecido. 0 questionário continha informações sobre a criança (sexo, idade e cor), o cuidador (sexo, escolaridade e parentesco), o motivo da não ida a UBS e a unidade de referência (bairro e UBS). A segunda etapa da coleta de dados foi realizada nas fichas de atendimento, após o término da consulta, sendo coletadas as queixas principais descritos pelos médicos e os diagnósticos clínicos estabelecidos. A pesquisa foi conduzida de acordo com os padrões éticos exigidos.

Para análise dos dados foram aplicados procedimentos da estatística descritiva, com avaliação da frequência e percentual, não sendo realizado nenhum teste estatístico. Os resultados foram apresentados por meio de tabelas e a análise foi realizada com auxílio do Software Stata 10.0.

\section{Resultados}

Nos seis meses em que decorreu o estudo, 254 pacientes pediátricos e seus cuidadores, que procuraram atendimento médico no PAI, durante o horário de expediente das Unidades Básicas de Saúde, fizeram parte da amostra deste estudo. Destes, 7 foram excluídos por não ter sido localizado o prontuário perfazendo uma amostra final de 247 sujeitos. Observa-se na tabela 1 que a maior parte das crianças eram do sexo feminino $(53,4 \%)$, possuíam menos de 5 anos de idade $(62,3 \%)$ e eram da cor branca (78,5\%).As características dos cuidadores que acompanharam as crianças a consulta, estão na tabela 2 e mostram que a maioria eram mães $(82,2 \%)$, estavam na faixa etária adulta $(93,1 \%)$,possuíam cônjuge $(58,7 \%)$ e a escolaridade era menor que o ensino médico completo $(62,4 \%)$.

\begin{tabular}{|c|c|c|c|}
\hline & Característica & $\bar{N}$ & $\%$ \\
\hline & \multicolumn{3}{|l|}{ Sexo } \\
\hline & Masculino & 115 & 46,6 \\
\hline & Feminino & 132 & 53,4 \\
\hline & \multicolumn{3}{|l|}{ Faixa etária (anos) } \\
\hline & & 50 & 20,2 \\
\hline & $\begin{array}{c}<1 \\
1 \mid-2\end{array}$ & 29 & 11,7 \\
\hline & $2 \mid-5$ & 75 & 30,4 \\
\hline & $5 \mid-10$ & 73 & 29,6 \\
\hline & $\geq 10$ & 20 & 8,1 \\
\hline & \multicolumn{3}{|l|}{ Cor } \\
\hline & Branca & 194 & 78,5 \\
\hline & Negra & 14 & 5,7 \\
\hline & Parda & 39 & 15,8 \\
\hline
\end{tabular}


Tabela 2 - Características demográficas dos cuidadores dos pacientes pediátricos atendidos no pronto atendimento infantil municipal de Santa Maria/RS

\begin{tabular}{ccc}
\hline Característica & $\mathrm{N}$ & $\%$ \\
\hline Parentesco & & \\
Mãe & 203 & 82,2 \\
Pai & 21 & 8,5 \\
Avó & 11 & 4,4 \\
Outros & 12 & 4,9
\end{tabular}

Faixa etária (anos)

\begin{tabular}{ccc}
$14-$ I19 & 13 & 5,3 \\
$19-$ I 59 & 230 & 93,1 \\
$\geq 60$ & 4 & 1,6 \\
Estado civil & & \\
Com cônjuge & 145 & 58,7 \\
Sem cônjuge & 102 & 41,3 \\
Escolaridade & & \\
Não alfabetizado & 2 & 0,8 \\
Fundamental incompleto & 77 & 31,2 \\
Fundamental completo & 41 & 16,6 \\
Médio incompleto & 34 & 13,8 \\
Médio completo & 83 & 33,6 \\
Superior & 4 & 4,0 \\
\hline $\mathrm{N}=$ número & &
\end{tabular}

As queixas mais frequentemente relatadas aos médicos foram: tosse (32\%), seguida de febre $(30 \%)$ como queixas isoladas ou associadas a outros sintomas. Quanto aos diagnósticos as afecções respiratórias foram responsáveis por $59,1 \%$ das consultas, sendo a infecção de vias aéreas superiores (42,9\%) as mais prevalentes (tabela 3). 
Tabela 3 - Principais queixas e diagnósticos verificados no pronto atendimento infantil municipal

\begin{tabular}{ccc}
\hline Variáveis & $\mathrm{N}$ & $\%$ \\
\hline Queixa principal & & \\
Tosse & 79 & 32,0 \\
Febre & 74 & 30,0 \\
Dor de garganta & 23 & 9,3 \\
Chiado & 17 & 6,9 \\
Vômito & 24 & 9,7 \\
Diarreia & 12 & 4,9 \\
Lesões de pele & 17 & 6,9 \\
Outras & 7 & 0,3 \\
Diagnóstico clínico & & \\
IVAS & 106 & 42,9 \\
Asma & 25 & 10,1 \\
Pneumonia & 15 & 6,1 \\
Gastroenterite & 20 & 8,1 \\
Outros & 81 & 33,8 \\
\hline número & &
\end{tabular}

Os motivos que determinaram a falta de procura das Unidades Básicas de Saúde para o atendimento médico (tabela 4) foram principalmente, a falta de fichas e de pediatra $(65,2 \%$ ) e preferência pelo atendimento no PAI $(18,2 \%)$.

Tabela 4 - Motivos referidos pelos cuidadores para não procurar a Unidade Básica de Saúde

\begin{tabular}{ccc}
\hline Motivos & $\mathrm{N}$ & $\%$ \\
\hline Falta de ficha & 110 & 44,5 \\
Não tem pediatra & 51 & 20,7 \\
Preferencia pelo PAI & 45 & 18,2 \\
Unidade fechada & 22 & 8,9 \\
Proximidade do PAI & 8 & 3,3 \\
Encaminhado pela UBS & 7 & 2,8 \\
Outros & 4 & 1,6 \\
\hline $\mathrm{N}=$ número & &
\end{tabular}

Em relação às unidades básicas de saúde de referência dos pacientes (tabela 5), cinco unidades (Centro Social Urbano, Floriano Rocha, Oneyde de Carvalho, Ruben Noal e Kennedy) são referência para aproximadamente $53,8 \%$ da população entrevistada, enquanto doze unidades com Estratégia de Saúde da família foi responsável por 23,1\% dos atendimentos. Quanto à procedência dos pacientes em relação às regiões administrativas, da Rev. Saúde (Santa Maria), Santa Maria, v.39, n.2, p.79-88, Jul./Dez.2013. central vieram $48,1 \%$ dos pacientes, seguidos pelos da região oeste $(36,5 \%)$ e norte $(13,8 \%)$. 
Tabela 5 - Unidades Básicas de Saúde de referência e região administrativa da residência dos pacientes atendidos no PAI de Santa Maria/RS.

\begin{tabular}{|c|c|c|}
\hline Característica & $\mathrm{N}$ & $\%$ \\
\hline \multicolumn{3}{|l|}{ Unidades Básicas de Saúde } \\
\hline Com ESF (12 UBS) & 57 & 23,1 \\
\hline Centro Social Urbano & 29 & 11,8 \\
\hline Floriano Rocha & 26 & 10,5 \\
\hline Kennedy & 27 & 10,9 \\
\hline Ruben Noal & 26 & 10,5 \\
\hline Oneyde de Carvalho & 25 & 10,1 \\
\hline Outros & 49 & 19,9 \\
\hline Não informado & 8 & 3,2 \\
\hline \multicolumn{3}{|l|}{ Regiões Administrativas } \\
\hline Centro & 70 & 48,1 \\
\hline Oeste & 90 & 36,5 \\
\hline Norte & 34 & 13,8 \\
\hline Sul & 24 & 9,7 \\
\hline Nordeste & 18 & 7,3 \\
\hline Distritos & 10 & 4,0 \\
\hline Outra cidade & 1 & 0,4 \\
\hline
\end{tabular}

\section{Discussão}

Neste estudo foram verificadas as características epidemiológicas e clínicas do atendimento pediátrico realizado no Pronto Atendimento Municipal Infantil de Santa Maria/RS, além dos motivos da sua procura, quem leva a criança para 0 atendimento e os locais onde residem.

Observou-se que houve predominância do sexo feminino em relação ao masculino com diferença de $7 \%$. Esta informação diverge não apenas de outros estudos,como também de dados do Censo de 2010 do Instituto Brasileiro de Geografia e Estatística (IBGE) do município que mostra que há $3 \%$ mais homens que mulheres nesta faixa etária no município².É possível que a prevalência,apresentada neste estudo, seja devido ao maior cuidado em relação às meninas, por serem estas consideradas mais frágeis e consequentemente levadas aos serviços em busca de atendimento com maiorrapidez $z^{9,10-14 .}$

Outro dado epidemiológico diz respeito à faixa etária que mais é levado ao PAl, onde

Saúde (Santa Maria), v.39, n.2, p.79-88, 2013. Pronto atendimento infantil: Quem usa e por que

motivo $62,3 \%$ dos pacientes eram lactentes ou pré-escolares. Este fato já tem sido observado em outros estudos, sendo, portanto, esta faixa etária mais predisposta a enfermidades que preocupam os cuidadores e requerem maior atenção do pediatra, mesmo que muitas vezes consideradas banais ${ }^{12-13,15-16}$. 
O sintoma de tosse foi à queixa mais frequente referida neste estudo, o que diverge de outros que mostram ser a febre como mais frequente ${ }^{6,10,17}$. No entanto, a diferença entre estas duas queixas foi de cerca de dois pontos percentuais podendo ser considerada mínima. Ressalta-se que o período de coleta de dados em que há mais meses considerados de inverno e primavera (julho-novembro) em relação ao verão (dezembro), pode explicar ser 0 sintoma de tosse tão prevalente.

Apesar de ter prevalecido o período de inverno/primavera ao verão na coleta de dados, os resultados encontrados para diagnósticos se assemelham a outros estudos, em que as doenças respiratórias, incluindo Infecção de Vias Aéreas Superiores, Asma e Pneumoniaforam prevalentes ${ }^{2,11-13}$. Por outro lado, as causas externas, como acidentes, que caracterizam muitas vezes urgência médica, tiveram baixa prevalência (menos de $5 \%$ ) e nem foram apresentadas nos resultados separadamente. Essa constatação entre estes dois dados demonstra que os responsáveis pelas crianças utilizam frequentemente serviços de urgência/emergência por causas clínicas. Assim, fica claroa maior frequência da utilização dos serviços de pronto atendimento em detrimento da Atenção Básica a Saúde, bem como denunciando, em alguns casos, a ausência de outros locais para atendimentos pediátricos nas proximidades da residência ${ }^{10,17-18}$.

Como a coleta de dados foi realizada no período em que as unidades básicas deveriam estar abertas (manhã e tarde), pode-severificar o real motivo que levam as pessoas a buscarem atendimento no PAI e quais as unidades com maiores problemas segundo os entrevistados, uma vez que nos demais horários haveria justificativa para o uso do PAI pela população6-9. Alguns estudos apontam que o maior uso de serviços de emergência está relacionado à menor distância da moradia ao serviço ${ }^{3,9,14,17-18}$. No entanto, nesta pesquisa, 0 percentual de entrevistados que relatou a proximidade ao PAI como motivo de busca pelo atendimento,não foi considerado relevante. $O$ destaque seria para a falta de fichas, que está relacionado à demanda e ao número de fichas disponíveis em quantidade insuficiente para suportá-la, situação já relatada como importante por outro estudo ${ }^{7}$. A falta de pediatra também foi relatada $(20,7 \%)$ mostrando que há ainda UBS com falta de profissionais adequados para atender a população. A preferência pelo PAI ocupa o terceiro lugar(18,2\%) demonstrando que ainda se relaciona perante a população uma imagem de melhor acessibilidade e resolutividade a este serviço, principalmente ligado a maior densidade tecnológica oferecida, além de certa desconfiança ao serviço prestado nas UBS 5-6,9-10,19.

Quando questionados a cerca da unidade básica de referência a maior parte dos entrevistados citaram unidades básicas que não possuem Estratégia de Saúde da Família (ESF). Isso provavelmente remete a que as primeiras têm maiores dificuldades em atender a população pediátrica e desta forma menor desempenho em comparação com as unidades que possuem ESF, fato já explicitado na literatura20-21.

A maior procura do PAI pela população que reside na região central se deve ao fato de que $22,9 \%$ da população de Santa Maria residem nesta área. No entanto, duas das cinco unidades mais citadas (Ruben Noal e Floriano Rocha) estão localizadas na Região Administrativa Oeste, sendo responsável por 36,5\% da população entrevistada e atendida no PAI. Esta porcentagem ultrapassa a população que reside na região (21,1\% segundo o IBGE) e pode estar relacionada a situação socioeconômica, uma vez que é a $3^{\mathrm{a}}$ região com a menor renda média e onde um menor número de pessoas recebem dez ou mais salários mínimos, além de possuir mais residências sem saneamento básico. Somado a isso, existe baixa escolaridade de seus habitantes, podendo-se inferir que existe desconhecimento sobre a

Rev. Saúde (Santa Maria), Santa Maria, v.39, n.2, p.79-88, Jul./Dez.2013. Santos, M. L.; et al. 
legítima função do Pronto Atendimento Infantil ou o que é considerado urgência por esta população ${ }^{3-4}$. Cabe ressaltar que esta é a região do município que possui o maior número de UBS com ou sem ESF e desta forma, a procura pelo pronto atendimento não pode estar associada a pouca quantidade de unidades básicas ofertadas nesta região ou o fato de estas estarem distantes das residências dos entrevistados como afirmam alguns estudos ${ }^{3-4, ~ 9, ~ 14-16 . ~}$

Os problemas que as UBS, em especial as que não possuem ESF enfrentam, como a falta de fichas e de profissionais que possam atender a maior parte das pessoas que buscam atendimento, gera na população certa desconfiança em relação aos serviços das unidades. Isto somado ao fato de que, historicamente, as Unidades de Pronto Atendimento já possuem imagem de resolutividade, confiabilidade e legitimidade pela população, acarreta uma busca maior pelas unidades de pronto atendimento ${ }^{5-6,9}$.Assim, pelo fato da maioria dos atendimentos realizados no PAI de Santa Maria/RS serem devido a problemas clínicos, devido ao desconhecimento da população da legítima função do pronto atendimento ou talvez associado a um melhor atendimento e, principalmente, pela dificuldade de acesso ao serviço de algumas UBS, pode-se perceber que o Pronto Atendimento Municipal Infantil do município é utilizado pela população como porta de entrada do sistema de saúde, como concluíram estudos em outras regiões brasileiras e países ${ }^{5,19} . \mathrm{E}$ ao ser utilizado desta maneira, pode sobrecarregar a equipe profissional fazendo com que a qualidade da assistência prestada tanto para aqueles que realmente necessitam de atendimento de urgência quanto às consultas eletivas, inadequadas a este serviço, que reflete a maioria, possa ficar comprometida, tal como elevar os gastos ${ }^{5-6}$.

\section{Conclusão}

Os resultados deste estudo permitem concluir que o perfil dos usuários atendidos no PAI durante o período tem distribuição semelhante em relação ao sexo, sendo crianças de até os 5 anos de idade, de modo especial os menores de um ano. Os pacientes são levados à consulta pelas suas mães, que são adultas jovens, com companheiro e com escolaridade menor que o ensino médio completo.

Os principais sinais apresentados pelas crianças foram tosse e febre, sendo as doenças respiratórias as mais prevalentes. Em relação ao motivo para não procura da Unidade Básica de Saúde prevaleceu à dificuldade de conseguir ficha para a consulta, seguida da falta de pediatra e da própria preferência dos usuários pelo PAI. A população que mais utiliza o serviço é a da região central e da região oeste da cidade.

Assim, as conclusões deste estudo sugerem a necessidade de readequar a atenção prestada à população infantil nas unidades básicas de saúde, para que se consiga um melhor modelo assistencial. A reeducação da população sobre os serviços prestados nas UBS, a reorganização da mesma e o esclarecimento do que realmente é urgência, podem contribuir para a solução de alguns dos problemas observados, possibilitando a mudança da realidade do Pronto Atendimento e tornando as Unidades Básicas de Saúde, realmente, portas de entrada ao sistema.

Saúde (Santa Maria), v.39, n.2, p.79-88, 2013. 


\section{Referências Bibliográficas}

1. Brasil. Ministério da Saúde, seção Cidadão - UPA 24 Horas, UPAS. Acesso em 10 mai. 2013. Disponível em: http://portal.saude.gov.br/portal/saude/area.cfm?id_area=1791

2. Caldeira T, Santos G, Pontes E, Dourado R, Rodrigues L. O dia-a-dia de uma urgência pediátrica. ActaPediatr Port.2006; 1(37): 1-4.

3. Suruda A, Burns TJ, Knight S, Dean JM.Health insurance, neighborhood income, and emergency department usage by Utah children 1996-1998.BMC Health Serv Res. 2005; 5(29): 1-6.

4. Bianco A, Pileggi C, Angelillo IF. Non-urgent visits to a hospital emergency department in Italy.Public Health. 2003; 117(4): 250-5.

5. Northington WE, Brice JH, Zou B. Use of an emergency department by nonurgent patients. AmJ EmergMed. 2005; 23(2): 131-7.

6.Haltiwanger KA, Pines JM, Martin ML. The Pediatric Emergency Department: A Substitute for Primary Care?Cal J Emerg Med. 2006; 7(2): 26- 30.

7. Caccia-Bava MCG, Pereira MJB, Rocha JSY, Martinez EZ. Pronto-atendimento ou atenção básica: escolhas dos pacientes no SUS. Medicina (RibeirãoPreto). 2011; 44(4): 347-54.

8. Paul SA, Reddy MC,DeFlitch CJ. A Systematic Review of Simulation Studies Investigating Emergency Department Overcrowding.Simulation, 2010; 86(8-9): 559-68.

9. Kovacs MH, Feliciano KVO, SarinhoSW, Veras AACA. Acessibilidade às ações básicas entre crianças atendidas em serviços de pronto-socorro. J Pediatr (Rio J). 2005;81(3):251-8.

10.Batistela S, Guerreiro NP, Rosseto EG.Os motivos de procura pelo Pronto Socorro Pediátrico de um Hospital Universitário referidos pelos pais ou responsáveis.Seminaciencbiolsaude. 2008; 29(2): 121-130.

11.Castro CGJ, Almeida MF, Yamada AT, Garbin W, Oliveira NA, Oliveira MSet al. Estudo do perfil da demanda do serviço de pronto-socorro do Hospital de Itaim Paulista, relatório. São Paulo: Secretaria Estadual de Saúde de São Paulo/Faculdade de Saúde Pública, Universidade de São Paulo; 2002.

12.Dubuc IF, Ferrari RAP. Adolescentes atendidos num serviço público de urgência e emergência: perfil de morbidade e mortalidade.RevEletrEnf. 2006; 8(2):250-8.

13.Salgado RMP, Aguero FCM.Perfil dos pacientes pediátricos atendidos na emergência de um hospital universitário. Pediatria (São Paulo). 2010; 32(2): 90-7.

14.Simons DA, Monlleo IL, Simons SA, Araújo Jr. JL. Adequação da demanda de crianças e adolescentes atendidos na Unidade de Emergência em Maceió, Alagoas, Brasil.RevBrasSaudeMater Infant. 2010; 10(1): 57-67.

15.Goh AY, Chan TL, Abdel-Latiff ME.Paediatric utilization of a general emergency department in a developing country.ActaPaediatr. 2003; 92(8):965-9.

16. Martineau O, Martinot A, Hue V, Chartier A, Dorkenoo A, Guimber D. Effectiveness of a short-stay observation unit in a pediatric emergengy department. ArchPediatr. 2003; 10(5): 410-6.

17. Jacobs PC, Matos EP. Estudo exploratório dos atendimentos em unidades de emergência em Salvador Bahia. RevAssocMed Bras.2005; 51(6): 348-53.

18. Cabral APS, Souza WV. Serviço de Atendimento Móvel de Urgência (SAMU): análise da demanda e sua distribuição espacial em uma cidade do Nordeste brasileiro.RevBrasEpidemiol. 2008; 11(4): 530-40.

19. Puccini PT, Cornetta VD. Ocorrências em pronto-socorro: eventos sentinela para o monitoramento da atenção básica de saúde.Cad saúde pública. 2008;24(9):2032-2042.

Rev. Saúde (Santa Maria), Santa Maria, v.39, n.2, p.79-88, Jul./Dez.2013

20. Facchini LA, Piccini RX, Tomasi E, Thumé E, Silveira DS, Siqueira FVet al. Desempenho do PSF no Sul e no Nordeste do Brasil: avaliação institucional e epidemiológica da atenção básica à saúde. Ciênc saúde ${ }_{\text {ISSN }}^{2236-5843}$ coletiva, 2006; 11(3):669-81. 
21. Elias PE, Ferreira CW, Alves MCG, Cohn A, Kishima V, Escrivão Jr Aetal.Atenção Básica em Saúde: comparação entre PSF e UBS por estrato de exclusão social no município de São Paulo.Ciêncsaúde coletiva.2006; 11(3): 633-641.

\section{Marina Lucca Santos}

Endereço de Correspondência - Av. Prefeito Evandro Behr, 6705, apto 401. Bairro Camobi, CEP 97110-620, Santa Maria, RS, Brasil

Currículo Lattes: http://lattes.cnpq.br/0869889568905775

E-mail: I.marinasantos@gmail.com

Recebido em 24 de maio de 2013.

Aprovado em 23 de setembro de 2013. 